\section{Mortality in hospitalized older adults associated with Clostridium difficile infection at a district hospital}

\author{
Johannis Andreas Karas, ${ }^{1,2}$ \\ Simon Bradshaw, ${ }^{2}$ Wabbas Mahmud, ${ }^{2}$ \\ David A. Enoch ${ }^{1}$ \\ 'Clinical Microbiology \& Public Health \\ Laboratory, Health Protection Agency, \\ Papworth Hospital, Cambridge, UK; \\ ${ }^{2}$ Hinchingbrooke Hospital, Hinchingbrooke \\ Health Care NHS Trust, Huntingdon, UK
}

\begin{abstract}
Clostridium difficile is the most common cause of hospital acquired infectious diarrhea in the developed world and has re-emerged in recent years with apparent greater morbidity and mortality. Despite this, there is little recent published data from the UK concerning associated mortality. We performed a case control study at a UK district general hospital of 66 hospitalized patients over the age of 65 years with $C$. difficile infection compared to 3-5 controls matched for age, sex and minimum length of stay. We found a significant excess mortality of $11.5 \%$ at seven days, $26.2 \%$ at 30 days, $38.1 \%$ at 90 days and $41.4 \%$ at 180 days. C. difficile infection in hospitalized elderly patients may contribute to long-term mortality or be a marker of poor prognosis and cases may require more intensive long-term follow up to improve mortality.
\end{abstract}

\section{Introduction}

Clostridium difficile is the most common cause of hospital acquired infectious diarrhea in the developed world and has re-emerged in recent years with apparent greater morbidity and mortality. The re-emergence of $C$. difficile is believed to be partly due to the appearance of a more virulent strain and due to aging of the population. Reported mortality rates of $C$. difficile infection (CDI) in the United States increased from 5.7 per million in 1999 to 23.7 per million in 2004; the median age of death was 82 years. ${ }^{1}$ In the UK the Department of Health (DH) instituted mandatory surveillance of $C$. difficile infections and of deaths due to CDI. This has shown a preponderance of elderly patients, with $81 \%$ of reported $C$. difficile cases in England being over the age of 65 and $30 \%$ over 85 . The number of death certificates mentioning $C$. difficile infection in the UK also increased by $72 \%$ between 2005 and 2006 , although this could partly be explained by improved reporting. ${ }^{2}$ Published mortality data due to CDI in the UK is rare with only some small outbreak related studies in the 1990 s. $^{3.5}$ It is also difficult to determine the true attributable mortality for CDI in the absence of severe features such as pseudomembranous colitis as these patients are frequently frail and elderly with other co-morbidities. We performed a retrospective case control study of hospitalized patients over the age of 65 to determine the mortality associated with CDI at our district general hospital in the UK.

\section{Materials and Methods}

\section{Study population}

Hinchingbrooke Hospital is a district general hospital with acute medical, surgical (general \& orthopaedic), elderly care, women's services and intensive care wards. It has 266 beds and covers a predominantly semi-rural population of 150,000 people in Cambridgeshire with 30,602 annual admissions in 2007/8.

\section{Materials}

This case control study was performed as part of an audit to compare our rates of mortality with those published by Pepin et al. ${ }^{6}$ Cases of $C$. difficile in patients over 65 years of age between $1^{\text {st }}$ March 2007 and $29^{\text {th }}$ February 2008 were identified from our laboratory information system. Cell cytotoxicity testing was used to detect $C$. difficile throughout the study period. Specimens were tested if grade 5-7 according to the Bristol Stool Chart. ${ }^{7}$ Strain typing was only performed if features of severe disease were present or an outbreak was suspected, in line with national UK guidelines. Duplicates occurring within 30 days were ignored and the first sample retained. Duplicates occurring after 30 days were considered a recurrence. Community acquired CDI was defined as that occurring within 48 hours of admission, if the patient was not transferred from another hospital, whereas hospital acquired CDI was after this period. Community-onset CDI was defined as healthcare-associated if recovered within 48 hours of hospital admission from a patient who had not previously been admitted to hospital been admitted to hospital in the preceding three months. Community acquired cases were not case matched. Demographic, admission and discharge data were obtained from the hospital computerized medical notes system. Charlson co-morbidity scores were obtained for the cases but not the controls from the medical notes.

Admission and discharge dates for the cases
Correspondence: J Andreas Karas, Clinical Microbiology \& Public Health Laboratory, Health Protection Agency, Papworth Hospital, Papworth Everard, Cambridge CB23 2RE, UK

E-mail: andreas.karas@papworth.nhs.uk

Key words: Clostridium difficile, mortality.

Acknowledgments: we thank Marlis Emery and Heidi Eagle at Hinchingbrooke NHS Trust for assistance with data collection.

Contributions: JAK, conceived the project; JAK, SB and WM planned the study and did data collection. All contributed to data analysis and final write up.

Financial support: this study was supported by the Health Protection Agency and Hinching brooke NHS Trust.

Conflict of interest: the authors report no conflicts of interest.

Received for publication: 27 April 2010.

Revision received: 4 June 2010.

Accepted for publication: 4 June 2010 .

This work is licensed under a Creative Commons Attribution 3.0 License (by-nc 3.0).

(C) Copyright J.A. Karas et al., 2010

Licensee PAGEPress, Italy

Infectious Disease Reports 2010; 2:e8

doi:10.4081/idr.2010.e8

ranged between October 2006 and May 2008 . Controls were therefore selected from the period $1^{\text {st }}$ October 2006 to $31^{\text {st }}$ May 2008 from the hospital admissions database representing all hospital admissions and discharges in the selection period, and matched to cases for age at admission, sex and length of stay (LOS). Length of stay for the controls had to be at least the LOS until $C$. difficile positive of the case in each instance. Any individual control patient could only act as a control once.

Three to 5 controls were matched per case; where the available pool was greater, controls were selected by proximity to admission date of the case. Where the number of controls available was fewer than 3 by this method, the admission age was extended to \pm 1 year in order to select further controls.

\section{Analysis}

For statistical analysis, proportions were compared using Fishers exact test. Attributable mortality for days 7, 30, 90 and 180 were calculated as the difference between cases and controls at each time period. A Kaplan-Meier plot compared time to death for cases from date of first positive $C$. difficile test and for controls, day 0 was the day they reached the same interval since admission as their 


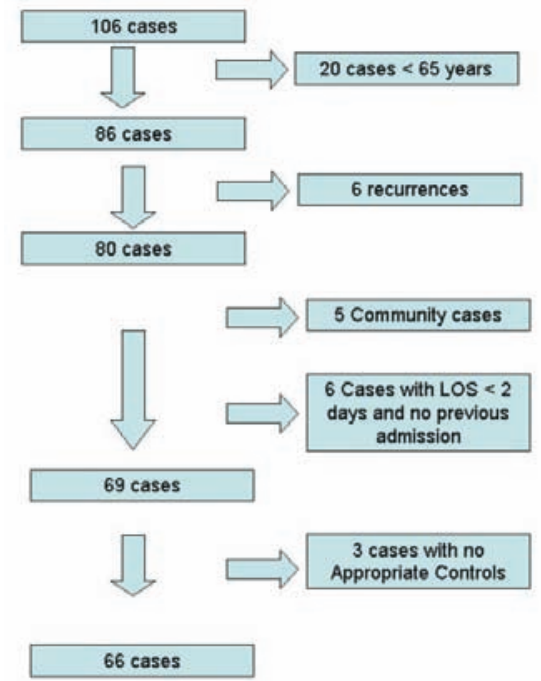

Figure 1. Flowchart of $C$. difficile cases and those excluded.

matched case. Ethical approval was sought but not required. The study was approved by Hinchingbrooke NHS Trust audit committee.

\section{Results}

A total of 106 episodes occurred in the study period. The rate of $C$. difficile infection was 52.2 cases per 100,000 population over the time period. Twenty occurred in the under 65 age group, of whom 3 died within 90 days. These were not further studied. Eighty-six episodes occurred in 80 patients over the age of 65 ; there were 6 (7.5\%) recurrences (Figure 1). Five cases were community acquired and never admitted, 6 were community onset $C$. difficile with no prior recent admissions; these 11 community acquired cases were excluded. This gives a hospital based rate of CDI among over 65 -year old patients of 24.4 per 10,000 bed days. It was not possible to find appropriate controls for 3 cases and they were excluded, leaving a total of 66 cases for matching and analysis. Two other cases yielded fewer than 3 controls in total, but were kept in the analysis due to having at least one appropriate control.

The crude mortality and cumulative associated mortality at days 7, 30, 90 and 180 days are shown in Table 1. Period specific associated mortality was $11.5 \%$ in the first seven days, $19.2 \%$ for days $8-30,25.0 \%$ for days $31-90$ and $16.4 \%$ for days $91-180$. A Kaplan-Meier plot of cases versus controls is shown in Figure 2.

The mean age of cases who died was 85.4 years and of survivors was 82.2 years $(\mathrm{P}=0.145)$. There was no significant difference in associated mortality between patients aged $65-79$ and those aged $80-89$, but this became significant in patients aged 90 years

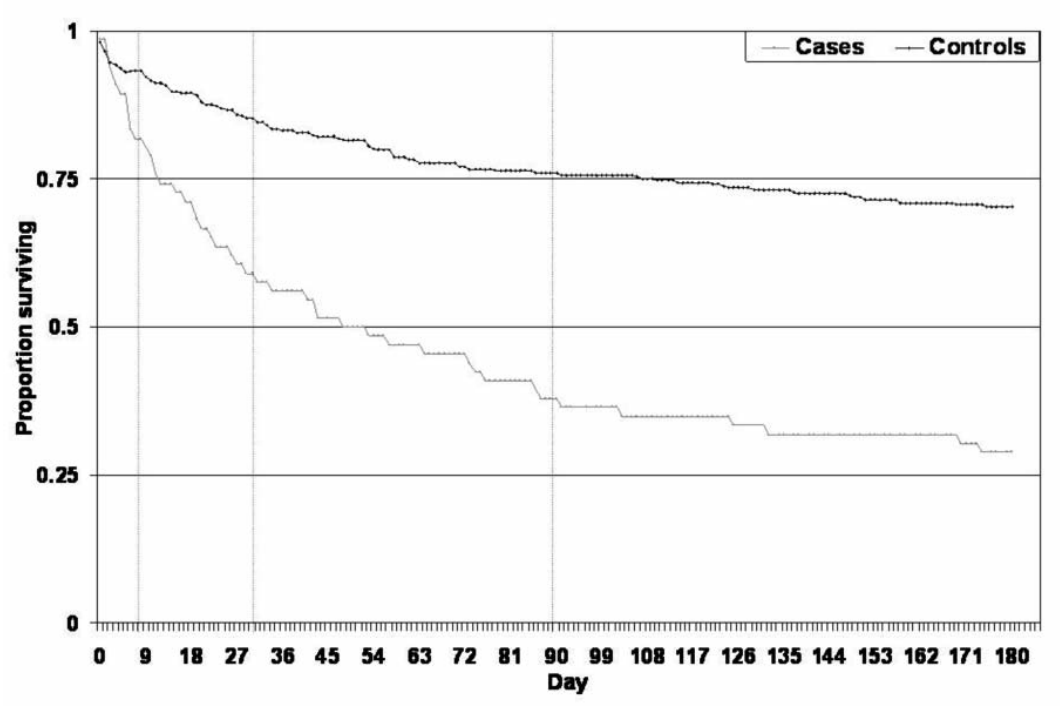

Figure 2. Kaplan-Meier Plot of survival to 180 days for $C$. difficile infection cases and controls. ${ }^{*}$ Calculated from day of first positive $C$. difficile test for cases and the time since reaching same point in admission for controls. Vertical lines are for days 7, 30 and 90.

Table 1. Attributes of cases and controls.

\begin{tabular}{lcccc} 
& Cases $(\mathrm{N}=66)$ & Controls (N=312) & P & Associated mortality \\
Mean age & 84.2 & 83.9 & & \\
Median age & 84.0 & 84.0 & & \\
\hline Sex (\% Male) & $33.3 \%$ & $32 \%$ & & \\
7 day mortality & $18.2 \%$ & $6.7 \%$ & 0.0137 & $11.5 \%$ \\
\hline 30 day mortality & $37.9 \%$ & $14.7 \%$ & $<0.001$ & $26.2 \%$ \\
90 day mortality & $59.1 \%$ & $24.0 \%$ & $<0.001$ & $38.1 \%$ \\
\hline 180 day mortality & $68.2 \%$ & $29.8 \%$ & $<0.001$ & $41.4 \%$ \\
\hline
\end{tabular}

and over among which only one of 14 cases was still alive at 90 days $(\mathrm{P}=0.009)$. Two patients required admission to Intensive Care. No patients required a colectomy. There was no significant difference when comparing the co-morbidity score of cases who died with those who survived, with $65 \%$ of deaths and $58 \%$ of survivors having a Charlson co-morbidity score of 1-3.

Very few isolates were ribotyped during the study period; however, ribotype 027 was isolated from 2 patients when a sample of isolates was tested in a UK national surveillance scheme.

\section{Discussion}

Published mortality data due to CDI in the UK is rare. Only three studies describe attributable mortality in UK hospitals and all are from the 1990s. ${ }^{3-5}$ Significant changes in epidemiology of $C$. difficile infection have occurred since then. Our hospital had a relatively low rate of CDI; it had the fourth lowest rate when compared to the other 17 acute hos- pitals in the East of England region. Over the study period, the incidence of CDI among all age groups in our population was 52.2 cases per 100,000 population which is much lower than that reported by Pepin et al. ${ }^{10}$ of 156.3 per 100,000 in 2003 during a major epidemic chiefly among hospitalized elderly people. We still, however, found a significant associated excess mortality in our patients aged over 65 of $11.5 \%$ at seven days, $26.2 \%$ at 30 days, $38.1 \%$ at 90 days and $41.4 \%$ at 180 days.

In a Canadian case control study (controls matched for age, sex, co-morbidity and length of stay), attributable mortality was $16 \% 30$ days after diagnosis, $17.6 \%$ three months after diagnosis and $21.4 \%$ six months after diagnosis. ${ }^{6}$ The attributable mortality at 30 days also increased with age, being $3.2 \%$ in the under 65 years, $14.3 \%$ in $65-74$ years and $19.7 \%$ in the over 75 years age groups. The mean age of CDI patients was 77.5 years. There was a corresponding increase in length of stay by an average of 10.7 additional days. We used this paper in our initial audit to compare our associated mortality. In our study, the median age was 84.2 which was 6.7 years higher, which may be a result of the study being limited to patients 
over 65 . They chose a cut-off of 72 hours to define hospital-acquired cases. We were also unable to do co-morbidity matching and this may have resulted in more healthy controls being selected, though we did match for minimum length of stay to mitigate this.

A 6-month prospective surveillance study in 2004-5 showed an overall attributable mortality of $5.7 \%$. The highest attributable mortality was in Quebec with $14.9 \%$ and was significantly higher in the over 65 years age group. ${ }^{8} \mathrm{~A}$ prospective study conducted in 2004 looked at cases of nosocomial CDI. ${ }^{9}$ The median age of cases was 76 and the 30-day attributable mortality was $6.9 \%$. Mortality increased with age with the highest 30-day attributable mortality being $14 \%$ in cases over 90 years.

There is much heterogeneity among published studies on measuring mortality in terms of definitions, patient groups, type and quality of study, information collected and information available. It appears that there has been an increase in incidence and mortality in recent years. ${ }^{10}$ The most frequently cited reason is the emergence of a hyper-virulent new strain (ribotype 027) that is able to produce significantly more toxin than previously known strains. ${ }^{11}$

Several studies also suggest that patients acquiring $C$. difficile are getting older, ${ }^{9,10,12}$ which is clearly a source of bias and may account for some of the increase in mortality. Loo et al. observed a greater than 5-fold increased incidence in patients over 80 versus those under $50,{ }^{9}$ while $89 \%$ of patients were over 65 years-old in another study. ${ }^{6}$ US data also showed a 5 -fold higher incidence in patients over 65 years of age. ${ }^{12}$ Gravel et al. showed that the median age of patients increased from 68 in 1997 to 73 in 2004/5. Mortality of patients over 90 years in our study was very high.

A recent study of death certificates in the US showed an increase in CDI-related mortality. CDI was reported as a cause of death for 20,642 persons from 1999-2004 in the USA and was the underlying cause of death for $59 \%$ of these deaths. The authors conclude that certain comorbid conditions are associated with CDI and that this contributes to the poor overall prognosis. ${ }^{13}$ Another study has suggested that CDI may be a marker for a critically ill patient population. ${ }^{14}$ The high crude mortality in many of the studies $\left(56 \%,{ }^{15} 55 \%,{ }^{13} 37 \%{ }^{6}\right)$ supports the concept of CDI being a prognostic marker for poor outcome. The crude mortality in our study of $37.9 \%$ at 30 days and $59.1 \%$ at 90 days is similar and supports the concept that CDI is a marker of poor overall prognosis.

Weaknesses of our work include its retrospective nature, relatively small sample size and single center setting. It was not possible to match sufficient controls for every case with advanced length of stay and we were unable to perform co-morbidity scoring on our controls. We excluded patients under the age of 65 as our local experience and the published literature indicate low complication rates in this group. Strain typing was infrequently performed as we adhered to Health Protection Agency criteria. However, we believe the data in this study is still of great value as it provides information about the associated mortality in a typical UK district hospital.

\section{Conclusions}

We have shown a large excess mortality associated with CDI in patients aged over 65 at our hospital which continues to rise to over $40 \%$ at 180 days after the initial positive sample. This suggests that CDI either contributes to long-term mortality or is a marker of poor prognosis. CDI patients may require more intensive long-term follow up if mortality rates in the elderly are to be improved. We support the need for further prospective multi-center studies to more accurately describe this excess mortality.

\section{References}

1. Redelings MD, Sorvillo F, Mascola L. Increase in Clostridium difficile-related mortality rates, United States, 1999-2004. Emerg Infect Dis 2007;13:1417-9.

2. Creagh H. Number of deaths due to C.difficile rose by $72 \%$ in one year. BMJ 2008 ; 336:529.

3. Cartmill TD, Panigrahi H, Worsley MA, et al. Management and control of a large outbreak of diarrhoea due to Clostridium difficile. J Hosp Infect 1994;27:1-15.

4. Impallomeni M, Galletly NP, Wort SJ, et al. Increased risk of diarrhoea caused by Clostridium difficile in elderly patients receiving cefotaxime. BMJ 1995;311:1345-6.

5. Lesna M, Parham DM. Risk of diarrhoea due to Clostridium difficile during cefotaxime treatment. Mortality due to $\mathrm{C}$ difficile colitis in elderly people has been underestimated. BMJ 1996;312:778.

6. Pepin J, Valiquette L, Cossette B. Mortality attributable to nosocomial Clostridium difficile-associated disease during an epidemic caused by a hypervirulent strain in Quebec. CMAJ 2005;173:1037-42.

7. Lewis SJ, Heaton KW. Stool form scale as a useful guide to intestinal transit time. Scand J Gastroenterol 1997;32:920-4.

8. Gravel D, Miller M, Simor A, et al. Health care-associated Clostridium difficile infection in adults admitted to acute care hospitals in Canada: a Canadian Nosocomial Infection Surveillance Program Study. Clin Infect Dis 2009;48:568-76.

9. Loo VG, Poirier L, Miller MA, et al. A predominantly clonal multi-institutional outbreak of Clostridium difficile-associated diarrhea with high morbidity and mortality. N Engl J Med 2005;353:2442-9.

10. Pepin J, Valiquette L, Alary ME, et al. Clostridium difficile-associated diarrhea in a region of Quebec from 1991 to 2003: a changing pattern of disease severity. CMAJ 2004;171:466-72.

11. Warny M, Pepin J, Fang A, et al. Toxin production by an emerging strain of Clostridium difficile associated with outbreaks of severe disease in North America and Europe. Lancet 2005;366:1079-84.

12. McDonald LC, Owings M, Jernigan DB. Clostridium difficile infection in patients discharged from US short-stay hospitals, 1996-2003. Emerg Infect Dis 2006;12:40915.

13. Changela U, Cannon JP, Aneziokoro C, et al. Risk factors and mortality associated with Clostridium difficile-associated diarrhoea a a VA hospital. Int $\mathbf{J}$ Antimicrob Agents 2004;24:562-6.

14. Dallal RM, Harbrecht BG, Boujoukas AJ, et al. Fulminant Clostridium difficile: an underappreciated and increasing cause of death and complications. Ann Surg 2002; 235:363-72.

15. Bishara J, Peled N, Pitlik S, et al. Mortality of patients with antibiotic-associated diarrhoea: the impact of Clostridium difficile. J Hosp Infect 2008;68:308-14. 